\title{
PERSPECTIVAS DE JUVENTUD EN EL IMAGINARIO DE LA POLÍTICA PÚBLICA
}

\author{
KERSTIN HEIN* \\ ANA CÁRDENAS ${ }^{* *}$
}

\begin{abstract}
RESUMEN
El presente artículo da cuenta de algunos de los resultados obtenidos a partir de una investigación que tiene como objetivo central generar un marco conceptual que contribuya a la descripción y el análisis de políticas locales de inclusión social a favor de la juventud en Santiago. El material que se presenta fue elaborado a partir de entrevistas realizadas a expertos del área y corresponde a un análisis y discusión de conceptos de juventud presentes en la política pública nacional. En cuanto a los resultados se plantea la existencia de una mirada que establece una fuerte asociación entre el ser joven y el ser estudiante y se discute lo que esto significa en términos de políticas de juventud. Posteriormente se analizan algunos matices que adquiere el concepto de juventud en la política pública lo que incluye una discusión acerca de los criterios que definen al individuo joven, la perspectiva del jove2 mo sujeto de derecho, el hecho de que los jóvenes son más bien objetos que sujetos de la política pública y el problema de no ser considerados clientes del Estado. Finalmente se trata de arrojar luces sobre la vinculación que los jóvenes establecen con respecto a la política pública.

\section{PALABRAS CLAVE: CONCEPTOS DE JUVENTUD, POLÍTICAS DE JUVENTUD, PARTICIPACIÓN}

* Psicóloga y doctora en psicología social y sociología. Investigadora Asociada de la Escuela de Psicología de la Universidad de Santiago de Chile. E-Mail: kerstin.hein@usach.cl.

** Socióloga y doctora en sociología. Académica de la Escuela de Sociología de la Universidad Diego Portales. E-Mail: ana.cardenas@udp.cl.
\end{abstract}




\title{
PERSPECTIVAS DE JUVENTUDE NO IMAGINÁRIO DA POLÍTICA PÚBLICA
}

\begin{abstract}
RESUMO
O presente artigo traz alguns resultados de uma pesquisa cujo objetivo central era a criação de um marco conceitual que contribuísse para a descrição e análise de políticas locais de inclusão social da juventude em Santiago. O material aqui apresentado foi elaborado a partir de entrevistas realizadas com especialistas da área e corresponde à análise e discussão de conceitos de juventude presentes na política pública nacional. Os resultados indicam a existência de um olhar que estabelece uma forte associação entre ser jovem e ser estudante, discutindo-se o que isto significa em termos de políticas de juventude. Em seguida, são analisadas algumas matizes atribuídas ao conceito de juventude na política pública, o que inclui uma discussão sobre os critérios que definem o indivíduo jovem, a perspectiva do jovem como sujeito de direito, o fato de que os jovens são mais objetos do que sujeitos da política pública, e o problema de não serem considerados clientes do Estado. Finalmente, o artigo procura esclarecer a vinculação que os jovens estabelecem em relação à política pública.
\end{abstract}

PALAVRAS CHAVE: CONCEITOS DE JUVENTUDE, POLÍTICAS DE JUVENTUDE, PARTICIPAÇÃO

\section{THE PERSPECTIVE OF YOUTH IN THE IMAGE OF THE PUBLIC POLICY}

\begin{abstract}
The present article gives account of some of the results obtained from an investigation that's central objective is to generate a conceptual frame which contributes to the description and the analysis of local policies of social inclusion in favor of the youth in Santiago. The material that is presented here was made by interviews from experts in this specific field and corresponds to an analysis and discussion of present concepts of youth in the national public policy. As far as the results are considered, it is set out to find the existence of the considers that establishes a strong association between youth as young-beings and youth as students; it also discusses what this means in terms of youth policies. Later some nuances are analyzed that the concept of youth in the public policy acquires which includes a discussion about the criteria that define the young individual, the perspective youth with rights, the fact that the youth are rather objects than subjects of the public policy and the problem of not being considered members of the State. Finally, trying to shed light on the entailment which the youth establish with respects to the public policy.
\end{abstract}




\section{INTRODUCCIÓN}

EL PRESENTE ARTÍCULO PRESENTA algunos de los resultados parciales obtenidos en el marco del proyecto «Jóvenes e inclusión social: estudio de casos de políticas locales de inclusión social a favor de la juventud en el Gran Santiago» financiado por el Departamento de Investigaciones Científicas y Tecnológicas de la Universidad de Santiago de Chile. Este estudio tiene como objetivo central generar un marco conceptual que contribuya a la descripción y el análisis de las políticas locales de inclusión social a favor de la juventud en el Gran Santiago y posteriormente, en diversos contextos nacionales e internacionales. Este artículo en particular analiza y discute el material recogido a partir de las diversas entrevistas a expertos realizadas al inicio de este proyecto de investigación. A través de dichas entrevistas, junto con la revisión de fuentes secundarias, se buscó elaborar un marco conceptual adecuado al análisis de las políticas de juventud, así como identificar los casos a nivel local a estudiar. Sin embargo, este artículo pretende hacer desde ya una contribución al debate sobre las políticas de juventud en Chile, poniendo en este caso el foco en el concepto de juventud que subyace a dichas políticas públicas. Esto, debido a que la reconstrucción de la definición pública de dicho concepto permitiría analizar y explicar la organización de las políticas públicas a favor de la juventud y el concepto de inclusión social asociado a éstas.

\section{MIRADAS SOBRE LA JUVENTUD CHILENA}

Actualmente se pueden observar dos formas centrales y distintivas de concebir la juventud chilena, cuya distinción resulta concordante con la tensión entre lo moderno y lo popular que ha acompañado la cultura latinoamericana a lo largo de su historia y que en el caso de la Juventud decanta en las imágenes opuestas de lo juvenil como expresión de los procesos de modernización, por un lado, y lo juvenil como elemento marginal y peligroso por el otro lado. En el último caso se trata de una mirada estigmatizante y controladora que se dirige a los sectores juveniles populares (cf. Goicovic, 2000; Iglesis, 2005; Sandoval, 2002), mientras que en el primer caso se instala la imagen de un joven exento de problemas, integrado, dedicado a sus estudios y al tiempo libre, conformista y consumidor (Sandoval, 2002; Iglesis, 2002).

Por otro lado, y más allá de la distinción entre jóvenes populares y jóvenes estudiantes, se encuentra la asociación de lo juvenil al cambio 
social. Cada vez que ciertos sectores juveniles se han visto envueltos en momentos históricos de cambio social, éstos se han vuelto visibles en el espacio público y pasado a servir de referentes sociales para caracterizar al sujeto joven en general (Iglesis, 2005). Es así como durante la década de 1960 el joven universitario que participa en la revuelta social y movimientos revolucionarios juveniles pasa a constituir la imagen de lo juvenil durante esa época (Silva, 1999; Dávila, 1999).

El protagonismo juvenil comprometido con el cambio social se desplaza durante la década siguiente. Aparece la generación de los 80 compuesta principalmente por jóvenes populares de sectores urbanos que participan en movimientos contestatarios contra la dictadura (Sandoval, 2002). Ellos pasan a constituir los nuevos referentes del sujeto joven en Chile. El rol protagónico que los jóvenes populares juegan en la transición hacia la democracia es reconocido posteriormente por los primeros gobiernos democráticos de modo de que se habla de la existencia de una deuda social para con estos jóvenes (Silva, 1999; Iglesis, 2003; Dávila, 1999, 2008). Dentro de este marco se visualiza al joven como un sujeto dañado y se busca reparar ese daño (Iglesis, 2003).

La generación de los 90 motiva un nuevo desplazamiento del significado social atribuido a la juventud. Según Silva (1999) se trata de una generación que crece y transita por la democracia, lo que les permite mayor movilidad y libertad de expresión. Se les reconoce su condición juvenil y también la deuda pendiente del Estado para con los jóvenes. No obstante, también se los percibe poco comprometidos con el contexto social y político en que viven. Especialmente los jóvenes estudiantes son percibidos como una generación consumista individualista y apática. A su vez los jóvenes populares son estigmatizados como sujetos desviados y peligrosos.

En este escenario irrumpen finalmente los estudiantes secundarios a través de su revolución pingüina en el año 2006 lo que vuelve a poner sobre la mesa la vinculación entre juventud y cambio social. El movimiento sorprende y cuestiona la idea de que los jóvenes «no están ni ahí», además de desplazar la imagen del sujeto joven hacia los sectores de educación secundaria.

\section{a) ¿Qué pasó con el joven popular?}

Según Goicovic (2000) los jóvenes populares han sido un grupo históricamente discriminado que ha transitado entre una integración laboral 
forzada y el control social por parte del Estado. Durante mucho tiempo resultaron invisibles ante los ojos de la opinión pública, pues no se los veía como sujetos sociales, sino que se los consideraba parte del mundo adulto. No obstante, esto cambia durante la década de 1980. La juventud popular adquiere visibilidad en el espacio público mediante su participación en los movimientos contestatarios y su rol protagónico en la restitución democrática del país (Silva, 1999; Sandoval, 2002). La mirada controladora que la sociedad chilena había dirigido hasta entonces hacia ellos es reemplazada por el reconocimiento de una deuda social para con los jóvenes.

A pesar de lo anterior a partir de los años 90 el discurso neoconservador en los medios provoca un nuevo desplazamiento del significado atribuido a los jóvenes populares (Iglesis, 2003) quienes pasan a ser estigmatizados y vinculados a problemas como la drogadicción, el alcoholismo, la delincuencia y el desempleo (Sánchez, 2000). La percepción del joven dañado se modifica y da paso a la percepción del joven popular como el prototipo de la juventud problema.

La progresiva desvinculación de los jóvenes populares de su anterior rol como agentes de cambio social y su consecuente desaparición como actores visibles de la escena nacional parece verse reflejada en la actual tendencia a reemplazar el concepto de juventud popular por el concepto de juventud vulnerable, lo cual refleja el cambio de significado atribuido a estos jóvenes.

Ahora yo tengo la sensación, cuando uno lee lo que se está escribiendo hoy en día sobre el mundo juvenil, yo tengo la sensación de que el concepto juventud popular ha ido perdiendo vigencia. Hoy en día no he leído cosas que hablen de los jóvenes populares, más bien se habla de jóvenes vulnerables, se habla de exclusión social, la inclusión-exclusión, entonces yo siento que es un concepto que ha ido como... como que fue un concepto de época, de la década de los 80. Lo que no quiere decir que los jóvenes pobres hayan desaparecido, siguen existiendo, y yo claramente hoy día les veo otro rol (Expertos CEJU, 30).

La desaparición de los sectores juveniles populares coincide con el aumento de la cobertura de la educación escolar y el consecuente aumento de los años que estos jóvenes permanecen en instituciones educacionales (INJUV, 2006). Es así como de manera progresiva se va imponiendo la asociación entre juventud y educación, y en particular para los jóvenes populares, entre juventud y educación secundaria. 


\section{b) Ahora todos deben ser estudiantes}

En general, no es raro encontrar una fuerte vinculación entre ser estudiante y ser joven. Posiblemente esto tenga relación con el hecho de que las sociedades modernas exigen mayores niveles de calificación de su población de lo que deriva una permanencia cada vez más prolongada de los jóvenes en los sistemas educativos y una postergación del ingreso al mercado laboral (Biggart et al., 2002). De este modo la educación se convierte, no sólo en una de las principales actividades del sujeto joven, sino que en una característica fundamental de la condición juvenil (Bendit, 2006). Algo similar plantea Baeza (2006) para el caso de Chile cuando afirma que el proceso de masificación de la Educación Secundaria ha contribuido al desarrollo de la juventud al aplazar el ingreso al mundo laboral, prolongar la dependencia familiar y en general retardar la asunción de responsabilidades vinculadas a la vida adulta.

La fuerte asociación entre juventud y educación tiene como consecuencia la percepción de que la vida cotidiana de los jóvenes debe encontrarse estructurada por el sistema educacional —ya sea escolar o universitario- y que el caso contrario constituye una desviación al ideal social (ibíd.).

En Chile, en la vida cotidiana, cuando uno conoce a un joven, que uno supone que está en edad escolar, la pregunta habitual que uno hace es ¿en qué curso estás?, es decir lo asumimos como parte de la vida cotidiana, y eso es un joven, un joven debe estar estudiando. A veces porque estamos viviendo en esto no nos damos cuenta, pero cuando uno está fuera del país uno se pregunta en qué curso está, en Haití por colocar un extremo, no resulta. Entonces hay ahí una primera cosa, y entonces claro, uno estadísticamente va a mirar y Chile tiene del orden de un 90\% de los jóvenes en edad escolar en enseñanza secundaria dentro del sistema escolar. Es cierto que, también, se ha ido produciendo un proceso mayor de masificación de la Educación Superior (Expertos CEJU, 36).

Una de las consecuencias que deriva de la consideración de que la educación y el estudio corresponden a la condición ideal del ser joven es la creencia de que el trabajo y la incorporación al mundo laboral es un tema adulto y no joven, con lo que los jóvenes trabajadores (que según la Quinta Encuesta Nacional de Juventud corresponden a aproximadamente el $40 \%$ de la población juvenil) corren el riesgo de caer en el olvido. Dentro de este marco de interpretación, la existencia 
del trabajo juvenil adquiere el significado de un «mal necesario». Existe la impresión de que los jóvenes trabajan cuando no tienen alternativa.

Por ejemplo, para mí, desde mi punto de vista, un sueño, una utopía; un joven entre 15 y 24 años no tiene porqué trabajar, no tiene porque estar en los índices de desempleo, desde mi punto de vista, un sueño país. Ese joven tiene que estar, tener la posibilidad de que el Estado le pueda entregar una educación de calidad, un perfeccionamiento y recursos para manutención para desarrollar diversas actividades; cosa que a los 23, 24 años esa persona tenga una inversión rica en capital humano, pueda salir al mercado laboral, y pueda aportar al desarrollo del país. No sé si me explico, es una utopía, y te lo planteo así, y para allá hay que ir trabajando (Experto INJUV, 68).

La asociación del concepto de juventud con el concepto de educación tiene entonces como consecuencia no sólo un concepto de juventud relativamente restringido, sino que impacta también el diseño y la implementación de políticas de juventud. Éstas estarán entonces principalmente dirigidas a resguardar la condición de estudiante de los jóvenes y menos interesadas en apoyar el empleo juvenil, lo que de hecho parece ser la tendencia en el diseño de programas dirigidos al sector juvenil durante los últimos años.

Y se han ido precisamente por una tendencia que viene hace un rato... siendo muy esquivo, el sujeto joven propiamente tal. Si uno lo agarra en esas tres subdivisiones, o subtramos etarios entre los quince y diecinueve años, eso es fundamentalmente, las políticas dirigidas supuestamente a jóvenes cada vez bajan más de edad, y son adolescentes, y cada vez son más población cautiva, son estudiantes (Experto CIDPA, 86).

Cabe hacer notar que la igualdad establecida entre el ser joven y el ser estudiante en el contexto chileno se encontraba inicialmente restringida al sector de los estudiantes universitarios (Dávila, 1999; Silva, 1999), mientras que en la actualidad podemos observar una progresiva, pero fuerte asociación entre educación secundaria y juventud. Esto incide sobre el diseño y la implementación de políticas públicas dirigidas hacia la juventud y en la institucionalidad encargada de tales tareas. De hecho se puede observar la tendencia a focalizar las intervenciones al ámbito del sistema de educación y un progresivo fortalecimiento del rol del Ministerio de Educación en el ámbito de la política juvenil. 
Focalizar la acción del Estado en el ámbito escolar tiene la ventaja de que permite elaborar acciones públicas sobre la base de una población cautiva, lo que facilita las intervenciones. Además garantiza un mayor alcance de los programas tanto educacionales como de otras áreas sociales (por ejemplo: salud) debido a la amplia cobertura de la enseñanza secundaria. Por otro lado, tal como señalábamos antes, tiene la desventaja de que deja fuera tanto a aquellos jóvenes cuya principal actividad es el trabajo como a aquellos jóvenes que pese a todos los esfuerzos no se encuentran integrados ni al sistema educacional ni al sistema laboral. Por lo demás también se corre el riesgo de terminar por reducir lo juvenil a lo escolar como de hecho ocurre en la ignorancia de la cultura juvenil por parte de la cultura escolar (Rodríguez, 2003; cf. Baeza, 2006). Esto implica una fuerte mirada adultocéntrica sobre la juventud basada en la disciplina y el control, lo que coloca al joven en una posición de obediencia pasiva y no de creación y participación activa.

\section{c) El derecho a pasarla bien}

La valoración de la juventud como etapa de estudio y preparación para la vida adulta se complementa con la idea de que se trata de una etapa de recreación y abandono al placer (Contreras, 2001). Se habla del derecho de los jóvenes a «pasarla bien», lo que contribuye mantener la visión de que los jóvenes son sujetos sin mayores responsabilidades (Baeza, 2006). Esto a su vez coloca a las políticas públicas ante la necesidad de incluir esta demanda en su oferta programática.

Desde el punto de vista de algunos de los expertos consultados, la oferta de actividades de tiempo libre y recreación constituye parte del desarrollo integral de un joven. Sin embargo, especialmente los sectores políticos más conservadores ven este tipo de acciones con cierto escepticismo.

Y una cuestión que nos cuesta mucho aquí en Chile, por nuestro conservadurismo y nuestra entropía, es el derecho a vivir la vida plenamente, y pasarla bien, que desde mi punto de vista es fundamental en la formación de un adulto, de un adulto mayor como tú; la dimensión cultural, la dimensión ocio, como tú la vives a plenitud para que tus años de juventud sean los mejores de tu vida, y que te marquen tu vida futura. No sólo en términos de ir a conciertos, obras de teatro, sino también en términos de calidad de vida en términos de prácticas deportivas, etc. 
Todo lo que involucra aquello, que te forjan valor para tu construcción como adulto, como adulto mayor que después tú la reproduces con las generaciones de descendencia. Ése es el concepto más claro, y eso nos ha costado mucho en nuestro país. O sea los recursos que yo me he conseguido asociado a cultura, recreación, rock, teatro, cine, en una política pública liderada por una clase antigua, por decirlo menos, y donde incluyo los de mi gobierno y los de afuera, ojo, los meto a todos en el mismo saco, cuesta entender que invertir en ocio, al final es una inversión que tiene rentabilidad en el mediano y largo plazo. Entonces en eso estamos trabajando (Experto INJUV, 35).

La identificación de lo juvenil y lo recreativo también genera dudas entre algunos expertos en temas de juventud, aunque no tanto frente a las actividades recreativas en sí, sino más bien con respecto al riesgo de reducir lo juvenil exclusivamente a lo recreativo. Es justamente ese temor el que aparece en las críticas realizadas a la creación del nuevo Ministerio de Juventud y Deportes.

No, yo creo que detrás está el hecho o la visión que mezclar juventud es sinónimo de deporte o cosas por el estilo, y que es una visión -incluso esta iniciativa - absolutamente irreflexiva (Experto CIDPA, 99).

La identificación de lo juvenil con la educación y el tiempo libre es concordante con la idea de que ser joven significa encontrarse en la condición de moratoria social, es decir en un período de preparación, experimentación, reflexión y espera, durante el cual el joven se encuentra liberado de asumir responsabilidades adultas hasta el momento en que cruce el umbral que separa el ser joven del ser adulto.

Sin embargo, esta concepción de juventud implica una noción lineal de la trayectoria biográfica lo que actualmente está siendo ampliamente discutido, fundamentalmente debido a los cambios socioculturales que se pueden observar en distintas partes del mundo y que afectan las transiciones a la vida adulta en varios sentidos. Entre estos cabe mencionar la ya señalada prolongación del período juvenil, la aceptación de la necesidad de aprendizaje continuo a través de todo el ciclo vital, la progresiva disolución de los límites entre lo que es ser adulto y lo que es ser joven, la des-estandarización e incluso reversibilidad de las trayectorias, etc. (Walther et al., 2002).

Los cambios en las trayectorias juveniles suponen entonces un cuestionamiento de la viabilidad del concepto de moratoria social, ya que éste representa una preparación para un mundo adulto cada vez 
menos cierto (Walther et al., 2002; Krauskopf, 2008). A su vez, implica una visión normativa de la juventud en que son los adultos quienes definen lo que se considera adecuado para los jóvenes (Sandoval, 2002).

Consecuentemente surge la necesidad de modificar los enfoques de juventud y de dejar de pensar en los sujetos jóvenes en términos de moratoria social, sino más bien de promover el desarrollo de su autonomía y su capacidad autorreflexiva. Esto significa, por un lado, no infantilizar a los jóvenes, reduciendo lo juvenil exclusivamente a la educación y el tiempo libre. Por otro lado, también implica dejar de lado la perspectiva adultocéntrica de que sólo el mundo adulto es valioso, no tomando con ello en serio a los jóvenes y sus necesidades particulares. En este sentido se vuelve cada vez más relevante complejizar los enfoques de juventud, otorgándole una mayor relevancia a la inclusión laboral juvenil y su compatibilización con los desafíos que impone la educación a lo largo de la vida, buscando con ello asegurar no sólo la participación simbólica sino que también material (Rodríguez, 2003) de las nuevas generaciones.

\section{JUVENTUD Y POLÍTICA PÚBLICA}

Hasta el momento hemos podido revisar y reflexionar sobre los principales ejes a partir de los cuales se construyen las diversas miradas sobre la juventud chilena y que tienen relevancia en el ámbito de la política pública del país. No obstante, se trata de una aproximación a la conceptualización del sujeto joven que no resulta exclusiva al ámbito específico de la acción pública, por lo que creemos que es necesario analizar más de cerca los distintos matices que adquiere el concepto de juventud en el imaginario de la política de juventud nacional.

\section{a) Definición de juventud desde la política pública}

Uno de los primeros aspectos que debemos considerar al analizar las perspectivas sobre juventud al interior de la política pública es la definición legal del sujeto joven, con lo cual ya nos topamos con el primer obstáculo y que es que en Chile no existe tal categoría. Ante este vacío legal el INJUV ha resuelto proponer una Ley de Juventud cuyo objetivo central es justamente llegar a definir al sujeto joven.

En Chile, la categoría Joven no existe por ley, no existe una ley de juventud. Existen los niños, los adolescentes, los adultos y los adultos 
mayores. El joven no existe, y para eso nosotros tenemos un borrador, que es una ley de juventud. Súper amplia, abstracta, que estamos esperando el momento propicio para dar el vamos (Experto injuv, 66).

Muy probablemente, la definición del sujeto joven se orientará según criterios de edad.

Los jóvenes en Chile son entre 15 y 29 años. No me gusta la definición de Naciones Unidas de 15 y 24 años, te digo de inmediato. Entre 15 y 29 años, eso es lo principal para iniciar el debate, bueno, qué son los jóvenes (Experto INJUV, 68).

La definición de Juventud mediante límites de edad es bastante común en el ámbito de las políticas públicas (Biggart et al., 2002; Bendit, 2006). Es así como las Naciones Unidas determina que el período de juventud se extiende desde los 15 hasta los 24 años de edad (OIJ, 2005). Por supuesto, esta norma no excluye el hecho de que cada país tenga reglamentaciones propias al respecto y de que incluso al interior de un mismo estado se establezcan diversos límites de edad para la categoría joven de acuerdo con las normas, intereses y necesidades de los diversos sectores. Para efectos del contexto chileno se ha logrado imponer la definición de los límites de edad propuesta por el INJUV que abarca a individuos desde los 15 hasta los 29 años de edad (INJUV, 2006).

A pesar de que resulta común recurrir a los límites de edad para definir lo que entendemos por juventud, se trata de una definición frecuentemente criticada por investigadores de juventud ya que resulta insuficiente, sobre todo si se considera que en sociedades modernas la condición juvenil parece extenderse a la vez que la transición a la vida adulta se vuelve cada vez más compleja e incierta (Biggart et al., 2002; Baeza, 2006; Bendit 2006). Lo anterior ha llevado a la necesidad de replantear y ampliar el tramo etáreo juvenil, ya sea hacia edades más tempranas o edades más tardías (Ravinet, 2008).

Una forma de resolver este dilema sería comprender la juventud como una fase del ciclo vital con características propias, es decir como una condición juvenil. El concepto de condición juvenil trasciende los límites de edad y permite reconocer a los jóvenes como sujetos autónomos que crean su propia cultura juvenil (Biggart et al., 2002; Baeza, 2006; Bendit, 2006). De acuerdo con esta perspectiva, si bien es cierto que el período de juventud necesariamente implica adquirir y desarrollar conocimientos y habilidades así como tomar importantes decisio- 
nes para la vida posterior, ya no se evalúa al joven exclusivamente a partir del ideal adulto, sino que se lo acepta como individuo pleno, lo cual resulta concordante con la visión del joven como un sujeto de derecho.

\section{b) Joven como sujeto de derecho}

La perspectiva del Joven como Sujeto de Derecho reconoce a los jóvenes como personas capaces de ejercer responsablemente sus derechos y libertades (OIJ, 2005). La perspectiva del joven como sujeto de derecho significa que los jóvenes adquieren derechos por el sólo hecho de ser jóvenes. De acuerdo a la opinión de los expertos, estos derechos deben ser garantizados por el Estado.

Es una cosa que puede salir hasta frase de campaña, en donde por el hecho de ser joven en nuestro país, el Estado tiene que hacerse cargo de un desarrollo integral de sus ciudadanos. En donde por ejemplo, el derecho a una educación de calidad, por el hecho de ser joven el Estado debe asegurarte eso. Por el hecho de ser joven, el Estado debe asegurarte un acceso a un empleo digno o decente. Por el hecho de ser joven, independiente que tenga contrato o no de trabajo, el Estado debe asegurarte una salud de calidad (Experto INJUV, 35).

El enfoque de derechos implica superar la actual tendencia a organizar las políticas de juventud en torno a necesidades. Esto no significa eliminar el enfoque de necesidades, pero sí lograr articularlo con un enfoque de derechos.

Yo creo que a un joven por un lado hay que ayudarle, que sea un beneficiario, darle oportunidades, más desde el punto de vista de las necesidades. En las metas del milenio también están más organizadas por necesidades que por derechos, y eso yo creo que todavía el enfoque es más de necesidades. Que es un enfoque que como te digo que hasta las Naciones Unidas con los objetivos del milenio lo plantean, o sea los niveles de ambos enfoques se articulen. Pero el enfoque de derechos es más exigente, entonces terminamos en compromisos más complicados (Experto FLACSO, 62).

El enfoque de derecho adquiere cada vez mayor relevancia en el debate público. Un ejemplo de aquello es la «Convención Iberoamericana de los Derechos de los Jóvenes» firmada en octubre del 2005 en Bada- 
joz (OIJ, 2005). Lamentablemente, y a pesar de reconocer la vigencia de la deuda social en relación con la población juvenil, el Estado chileno aún no ha ratificado esta convención.

\section{c) Joven como objeto, pero no como sujeto de la política pública}

Según Sánchez (2000) existen políticas para los jóvenes, políticas por los jóvenes y políticas con los jóvenes. Sólo en el último caso los jóvenes son consultados y llamados a participar en la formulación de políticas de juventud, mientras que en los dos primeros casos, las políticas públicas son diseñadas e implementadas desde el mundo adulto sin considerar mayormente la opinión de la gente joven. En otras palabras, el joven se constituye como un objeto de preocupación para la política pública, pero no participa —al menos no de manera regularcomo un sujeto activo en la formulación de la misma. De lo que se trata, entonces, es de dilucidar el diagnóstico que el mundo adulto realiza acerca de la juventud considerando no sólo lo que se plantea explícitamente, sino también lo que se omite acerca de los jóvenes, todo con el fin de lograr comprender la orientación de los programas de política de juventud.

i) Un diagnóstico que no considera la diversidad juvenil

El diagnóstico que realiza la política pública acerca de la juventud chilena tiende a desestimar su diversidad. Dicho de otra manera, a pesar de que se reconoce el valor de lo diverso y la necesidad de hablar de la existencia de «juventudes» más que de juventud como una sola (Iglesis, 2005), la política pública tiende a olvidar con facilidad la pluralidad de los jóvenes y a «meterlos a todos en el mismo saco». Es decir, existe la tendencia a tratarlos como una masa uniforme sin respetar la identidad propia de cada sujeto (Baeza, 2006).

Jóvenes y pobreza es igual drogadicción, por ejemplo. Pero cuando uno entra a sectores pobres, y empieza a entrevistar jóvenes, uno claramente encuentra a jóvenes que nunca han consumido drogas, que hay jóvenes que han experimentado con la droga, pero que se han salido, hay jóvenes que tienen un consumo muy esporádico, más bien de fin de semana, y que efectivamente hay jóvenes que están siendo muy consumidores. Y cuando uno trabaja con ellos, uno va descubriendo como su construcción de discurso es totalmente distinta, es decir el discurso de la impor- 
tancia del proyecto de vida, del sentido de vida, el no fallar en la familia, etc., que está muy presente en el joven no consumidor. El discurso del que experimenta, donde él quiere conocer la novedad, reconoce que los amigos presionan, etc., el discurso del consumidor de fin de semana es como si él sabe que está jugando con una cosa peligrosa, pero él cree que en cualquier momento la puede dejar. Las visiones son distintas, pero la política pública hacia el joven del sector pobre es una sola: el joven pobre es un joven drogadicto (Expertos CEJU, 38).

Al privilegiar la existencia de una sola juventud por sobre la existencia de múltiples juventudes, la política pública comete el error de ignorar a ciertos grupos de jóvenes como lo son las mujeres jóvenes, jóvenes provenientes de zonas rurales y jóvenes de origen étnico cuyas realidades cotidianas no se corresponden con la imagen dominante de juventud reflejada en la figura del hombre blanco, urbano y escolarizado.

Quizás valga la pena decir algo que yo creo que tienen que haber escuchado varias veces ustedes también, y es como la política trabaja con una construcción principalmente que si uno la empieza a profundizar, detrás de ella uno se da cuenta de que hay una visión de: joven, joven urbano y escolarizado, joven hombre. Es muy fuerte eso, y cuando hablamos de juventud, nos empezamos a dar cuenta de que sabemos muy poco de los jóvenes rurales, menos de los jóvenes indígenas y menos de la mujer joven indígena [...]. Entonces hay cuestiones de ese estilo, que nosotros como país nos ignoramos a nosotros mismos, es decir somos un país de blancos, sin indígenas, etc., y no conocemos lo que está pasando con el mundo indígena, somos un país urbano, no rural. Es cierto que proporcionalmente no son muchos, pero también desconocemos mucho, es decir todo nuestra construcción discursiva es de un joven urbano, hombre y principalmente escolarizado, escolarizado ya entrando en el mundo de la Educación Superior (Expertos CEJU, 43-45).

\section{ii) Una mirada fragmentada del individuo}

La mirada uniformadora de las políticas públicas no termina en la ignorancia de las diversas realidades juveniles que residen en nuestro país, sino que también parece desconocer la pluralidad de intereses y lógicas de acción de cada joven en sí.

Y es que hay una visión, a mi juicio, como él dice «uniformadora» del mundo juvenil desde las políticas públicas — de lo poco que hay-, que no reconoce una de las características básicas de la juventud actual, que 
es la diversidad, la heterogeneidad, la pluralidad y lógicas de acción. Son jóvenes plásticos, multifacéticos. Un mismo joven puede ser durante varias horas del día jugar roles distintos, entonces encasillarlos en una sola imagen me parece casi caricaturesco (Expertos CEJU, 40).

El desconocimiento de la plasticidad del individuo fragmenta la mirada que se dirige al joven: La política pública no ve al sujeto joven de manera completa y compleja, lo que en términos concretos significa que no logra elaborar una propuesta integral y articulada dirigida hacia los jóvenes.

Pero hay una tercera cosa que es también importante; en esta simplificación que de repente hacen de la juventud, miramos el joven escolar por un lado y, por otro lado, — por decirlo en términos sencillos- miramos a los jóvenes pokemones, pero a veces se nos olvida que el mismo pokemon es el mismo que está en clase en la mañana, que es el mismo que hiphopea, y que es el mismo que es punkie, etc. Es decir, hay en el discurso académico una claridad bastante más grande de que hoy en día la inteligencia no es una sola, que hay muchas inteligencias, las inteligencias múltiples. En el discurso académico hoy en día, hay muchas más aceptación también de que la construcción de la identidad es una construcción de identidades múltiples, que una persona que en la mañana es una cosa y en la tarde es otra, no es un esquizoide ni una cuestión patológica, sino que es una cuestión normal, pero eso la política pública no lo tiene muy incorporado, es decir, le es más fácil etiquetar al joven que es escolar y otros jóvenes que son de las tribus, cuando sabemos que son las mismas tribus que están al interior del colegio. Cuando uno está al interior de un colegio y mira, en un país como Chile, donde los jóvenes usan uniforme (a diferencia de otros países), al ojo de una persona no preparada, no ve diferencias, pero uno parado al lado de un joven, te dice: mira, ese es hiphop, por el pantalón, ese otro es de tal grupo, fíjate en el corte de pelo. Ese tipo de cosas, la política muchas veces simplifica, y no considera la diversidad que tiene el mundo juvenil (Expertos CEJU, 39).

\section{iii) Monólogo adultocéntrico: yo sé lo que tú necesitas}

En un análisis acerca del movimiento de los estudiantes secundarios, Baeza (2006) plantea de manera bastante categórica que buena parte de las políticas públicas se construye ignorando a los jóvenes. Lo dicho refleja una falta de diálogo entre el mundo juvenil y el mundo adulto en que los adultos hablan «de» los jóvenes pero no «con» los 
jóvenes. En otras palabras, los jóvenes pasan a ser objetos más que sujetos de la política pública. De esta forma las políticas de juventud elaboran una especie de monólogo en que se ve lo que se quiere ver y se ignora lo que realmente está pasando. La imagen de joven que se construye responde a una práctica institucional instalada más que a la real condición de la juventud chilena.

Entonces mi primer tema como acercamiento al sistema de la política pública, es cómo la política pública se construye a un joven que muchas veces no es el joven que existe en la realidad, sino que es un joven que le resulta funcional a lo que ya vienen haciendo. Entonces no leen desde los jóvenes, sino que lee desde ella misma, que es lo que gente como $\mathrm{C}$. B., L. Y. han señalado más de una vez como este adultocentrismo que existe en el presente (Expertos CEJU, 27).

De este modo las políticas públicas operan desde una lógica de autorreferencia, pero sin preguntarles a los jóvenes mismos. Se toman decisiones sobre y por los jóvenes, pero no desde ellos.

Normalmente las políticas de juventud son bastante unidireccionales, esto es, equipos del Estado que construyen las políticas, con muy poco diálogo hacia quienes van a ser los beneficiados, producto de estas mismas cosas que ya te señalaba. O sea, muchas veces esta visión de que yo sé lo que tú necesitas, con muy poco tiempo de escucha. Si uno lo mira por ejemplo en cosas de educación, se habla de los jóvenes, pero poco se habla con los jóvenes, lo que es distinto. Es decir, leer sobre la realidad juvenil, leer desde la realidad juvenil, exige una acción que es mucho más incómoda que hacer lo que se está haciendo, es decir, porque hay que salir de la posición en la que uno está, colocarse en la cuestión del otro, y desde el otro entenderla (Expertos CEJU, 56).

iv) Brecha entre el mundo juvenil y el mundo adulto

Los adultos suelen pensar que pueden comprender a los jóvenes a partir de su experiencia personal de juventud. No obstante, si bien todos los adultos han sido jóvenes alguna vez, no lo fueron en esta época en la cual los procesos de individualización y pluralización de los mundos de vida van haciendo de la juventud, o mejor dicho, de las juventudes actuales una experiencia distinta a la vivida por el mundo adulto (Baeza, 2006). Esto produce una falta de diálogo entre el mundo adulto y el mundo joven, lo que a su vez refuerza el monólogo 
adultocéntrico y dificulta la formulación de políticas públicas que realmente reflejen las necesidades de los jóvenes.

Entonces con las generaciones, hoy en día son otras las utopías sociales que se están formando los jóvenes y, además, la generación se ha ido cortando, es decir si antes las generaciones eran largas, podían durar décadas, hoy en día los jóvenes, es decir tú escuchas a hablar a los jóvenes de esta universidad (que generalmente son de pedagogía), dicen ellos que no entienden a los jóvenes. Es decir, cuando yo voy a hacer clases a ellos, yo no entiendo a los jóvenes, y hay una diferencia de edad entre ellos muy corta. No es ya la diferencia de padre a hijo. Entonces estamos frente a una falta de diálogo entre el mundo adulto y el mundo joven, donde el adulto construye desde su historia colectiva, desde su utopía, de su historia personal, o sea si nació en el estrato social tanto. Es decir, el esfuerzo de leer desde los jóvenes no es un esfuerzo que esté instalado, es un esfuerzo largo, complejo, y por lo tanto se simplifica con encuestas, con algunos estudios de baja calidad en relación a los distintos rigores, etc. [...] Bueno, muchas veces la falta de diálogo que hay en el mundo juvenil y el mundo adulto, es que el mundo adulto no conoce la historia colectiva de los jóvenes, sino que parte de su propia historia colectiva, y es una cosa que parte de las utopías sociales en las cuales fue formada, no las utopías sociales en las que han sido socializados los jóvenes (Expertos CEJU, 59).

La superación de la brecha generacional entre el mundo adulto y el mundo joven supondría encontrar espacios de comunicación e interacción mutua. Sin embargo, tanto la literatura especializada como los expertos entrevistados dan cuenta de lógicas de funcionamiento totalmente distintas entre jóvenes y adultos, lo que agudiza aún más las diferencias. Un claro ejemplo de esto lo constituye el ámbito de la política pública, en donde podemos presenciar la existencia de dos culturas participativas completamente diferentes entre sí: mientras que los adultos mantienen su concepto tradicional e institucional de participación, los jóvenes privilegian la vía informal y directa (Silva, 1999; Iglesis, 2005; Walther et al., 2002; INJUV, 2004), el vínculo con lo local, la organización en redes, la horizontalidad, la acción concreta y la democracia directa (Iglesis, 2005; Baeza, 2006).

Lo interesante es que la forma en que los jóvenes entienden la participación social se constituye y adquiere sentido en contraste con el concepto de participación propuesto por el mundo adulto. En este sentido los jóvenes justamente identifican como fortaleza que su for- 
ma de participación no funcione de acuerdo a las normas impuestas por la sociedad de adultos. Por su parte los adultos tienen dificultad en reconocer, entender y manejar la forma en que los jóvenes entienden la participación. Resulta inquietante el hecho de que no jueguen con las mismas reglas del juego (Contreras, 2001). Lo que queda finalmente tras este verdadero «choque cultural» (Baeza, 2006) es un complejo escenario de participación social.

\section{d) Jóvenes no son clientes del Estado}

A pesar de lo anteriormente dicho, reconocer que los jóvenes «sí están ahí» aunque «a su manera» es importante, pero no parece resolver el dilema de la falta de participación juvenil a través de los canales tradicionales, por lo menos no en términos de la dimensión juvenil en las políticas públicas. El problema radica en que al final de cuentas la reticencia hacia las instituciones políticas tradicionales sí afecta a los jóvenes, en el sentido de que dejan de ser clientes del Estado. De este modo los expertos consultados estuvieron de acuerdo en señalar que no resulta rentable para los actores políticos preocuparse por el tema juvenil, ya que no existe ganancia política para ellos. Para qué invertir en jóvenes, si con eso no se ganan votos.

¿Cuál es la gran pata coja, como se dice en nuestro país, de todo este modelo?: la participación de los jóvenes. En la medida de que la clase política vea que los jóvenes no son parte de la democracia, transversalmente te lo digo, la clase política no invierte en jóvenes. Si tienen que elegir entre un adulto mayor que vota, y un joven que no vota, y ahí vamos a llegar al tema del gobierno local, los recursos van acá. Y qué es lo que pasa con esto, se produce un círculo vicioso en donde el joven ve que no se invierte en él, y no se le habla a él, no participa en la democracia y esto se va reproduciendo (Experto INJUV, 30).

Claramente, los jóvenes no logran convertirse en un actor social de peso en el país, pues no acceden al poder social ni político para defender su punto de vista. Según los expertos entrevistados, esta situación se agudiza en Chile debido al gobierno militar, el que dejó como legado un sistema político rígido basado en el castigo y la obligatoriedad que ahuyenta a los jóvenes.

Pero el problema de Chile es un problema político importante en términos de redistribución de poderes, porque, y eso yo creo que lo mencioné 
ese día, yo más o menos lo digo ya, y lo dice mucha gente... Claramente el poder político está en un grupo etáreo mayor, y la dictadura dejó una herencia que permitía el control político al decir que la gente tenía que inscribirse para votar, o sea no era un poder natural Y si no votaba, era multada, por lo cual [...] la gente lo piensa mucho para inscribirse. Entonces, eso ha llevado a que los jóvenes no se inscriban, y hay estadísticas que es como el siete por ciento de jóvenes está inscrito (Experto FLACSO, 33).

Por otro lado, echar la culpa a los jóvenes tampoco resuelve el dilema. Algunos expertos señalan que los adultos también son parte del problema, ya que temen las consecuencias que podría tener una participación juvenil efectiva en el sistema político e intentan mantener la poca visibilidad de los jóvenes en la vida pública.

Nada, entonces es una realidad obvia que si hubiera la decisión de darle un lugar a los jóvenes en la vida política, los dos polos políticos apuntarían a eliminar la inscripción y dejarían el voto voluntario. Pero aunque las dos alas reconocen que eso es lo que se ocupa, nunca llevan a cabo la modificación. Ahora, eso ya es una señal muy clara, que no van a llevar a cabo esa modificación hasta que sea completamente necesaria. Necesaria en términos de metas políticas de estos grupos. Mientras puedan controlar las votaciones que están en una situación muy equidistante, se les desordena demasiado el naipe, metiendo a un grupo que no se sabe que va hacer. Entonces es una situación que yo creo que es un factor muy importante de lo que le pasa a los jóvenes aquí en Chile (Experto FLACso, 37).

En este sentido, lograr cambiar la situación de participación requiere un replanteamiento y redistribución del poder entre el sector adulto y el sector juvenil. Un importante avance en este sentido ha sido la aprobación en enero de 2009 de la modificación de la Constitución por parte del Senado lo que en el futuro permitirá la inscripción automática y el voto voluntario en Chile. Aún si no se logran implementar estas modificaciones para las elecciones presidenciales del presente año, los cambios prometen una mayor participación de los jóvenes en la política del país en el mediano a largo plazo.

\section{e) Vinculación de los jóvenes con la política pública}

Uno de los objetivos centrales de la investigación, cuyos resultados parciales se presentan en este artículo, es lograr dilucidar la vincula- 
ción de los jóvenes con la política pública. Si bien las entrevistas a expertos no estaban diseñadas para cumplir con este objetivo, en el transcurso de las entrevistas se plantearon algunas ideas interesantes con respecto a la percepción de la política pública y la forma en que los jóvenes se relacionan con los programas. Aún así no hay que olvidar que se trata de una mirada adulta sobre el comportamiento juvenil y que no son los jóvenes mismos quienes opinan al respecto.

i) Percepción de la política pública

A la luz de la opinión de los expertos, los jóvenes parecieran desconocer hoy la existencia de una oferta programática diseñada para ellos.

Es bastante, no es algo que no existe como tal. Pero cuál es el gran problema: que yo salgo afuera, y entrevisto a un chico, y yo le pregunto si el Estado le ayuda, él me va a decir que no hay política para mí (Experto INJUV, 39).

Por otro lado, aún en el caso de que exista conocimiento acerca de la existencia de una política pública para jóvenes, los jóvenes mismos no la perciben como una acción del Estado dirigida hacia ellos, sino que ven el beneficio como un servicio al cual ellos tienen derecho o bien como un servicio que ellos han obtenido gracias a su esfuerzo individual, todo lo cual resulta concordante con el discurso propio de la filosofía neoliberal en donde al estado le corresponde proteger la libertad individual (Sandoval, 2002) y con las exigencias del proceso de individualización en que «todo depende de uno» (Cárdenas, 2009). En el fondo, los jóvenes actuales perciben la política en términos más pragmáticos que idealistas y de acuerdo con sus propias posibilidades de logro individual (Sandoval, 2002).

Los jóvenes no tienen una opinión crítica frente al sistema escolar, es decir reconocen el tema de la diversidad, de la diferenciación, pero han incorporado con mucho esfuerzo también esa cosa del discurso de si yo logro algo no es el sistema, sino que soy yo entonces hay una responsabilidad muy alta sobre sí mismo, es decir, si yo me esfuerzo voy a entrar a la universidad, por último, mis papás no me pagaron un preuniversitario, y por eso no pude entrar a la universidad. En quinto o sexto lugar vendrán a decir que «yo estoy en una escuela pública que realmente era muy mala en calidad» (Expertos CEJU, 96). 


\section{ii) ¿Actores o consumidores?}

Desde el mundo adulto vinculado a la política pública frecuentemente se expresa molestia sobre la vinculación de tipo instrumental que algunos jóvenes establecen con la política pública y que resulta más cercana al comportamiento de un consumidor que de un actor social. Desde este punto de vista no es que los actores políticos no deseen incorporar a los jóvenes en la elaboración de políticas de juventud, sino que más bien son los jóvenes quienes no se interesan en participar en este proceso. La impresión es que los jóvenes consideran que la construcción de una política es responsabilidad de las instituciones públicas, mientras que ellos adoptan el rol del cliente que decide o no aceptar la oferta.

¿Quién puede desde una forma de interrelación o relacionamiento del gobierno local, a quien llama a ser protagonista, le interesa mucho eso de ser protagonista? Como dicen las chiquillas de C., co-constructor de políticas o planes de desarrollo juvenil en la expresión local. Eso me consta que es todo un proceso al respecto [...]. De lo divino y humano, pasando de lo que se te ocurra en eso. Nos estaban invitando al presupuesto participativo juvenil, y algunos representantes le dicen: no, a nosotros no nos interesa eso, eso es de responsabilidad de ustedes como municipio, ustedes decidan su inversión, ustedes vean, porqué nos meten en una cosa de atribución y que es de trabajo de ustedes. Entonces siempre lo hemos comentado de que es como contraejemplo, ya que todos dicen queremos participar en tomar decisiones y cuestiones. «No, nosotros no queremos, y después veamos si es que la oferta de ustedes... entonces empieza una lógica media de usuario, de cliente, es como veamos, cual es su oferta y veamos si nos interesa. Es un poco también con esas lógicas con que se mueven entre clientelares y usuarios, consumidores estos muchachos [...]. Y los muchachos también generan estrategias de desenvolvimiento en esos intersticios, también no son víctimas en ese sentido, y si no les gusta se llevan la pelota, lo que es muy recurrente. No pescan la invitación socializadora o el llamado a la participación que hacen, y eso tampoco es solamente de los jóvenes (Experto CIDPA, 106-108).

La actitud de consumidor desvincula a los jóvenes de las instituciones públicas. Si la institución pública no satisface las necesidades de los jóvenes, ellos se van a otra parte. No dependen de la institución pública, sino que aparecen como usuarios de sus servicios. 
Claro, y que me hablan de estos recitales de jóvenes que hacen para el Día Internacional de la Juventud, son tremendamente masivos. Todos los primeros viernes de octubre de cada año se hacen la fiesta de los mil tambores aquí, en Valparaíso. Son cabros de expresiones mas sociocultural, con batucadas, cualquier cuestión que meta bulla, baile y música. No sé, van tres mil, cinco mil cabros, y el elenco estable eran los artistas entre comillas, unos mil, y esos están todo el año, haciendo uso de su espacio, participando en su espacio concreto, que no tiene mucho que ver con el municipio ni el Consejo de la Cultura. El Consejo creo que les pasa una platita, pero bien simbólica, pero hay montones, en Valparaíso hay un montón de cabros que están haciendo regularmente sus asuntos, todas estas diversidades circenses, teatro, todas estas cuestiones, y hay cabros que están permanentemente haciendo esas cuestiones sin necesidad de tener ningún vínculo con el municipio o con la institucionalidad, con las instituciones, ni siquiera muchas veces por un sentimiento de oposición, o sea «no mira acá el municipio es no sé de qué partido, demócrata cristiano, y yo la verdad es que no, ando por allá, o es UDI o PC, no, ni siquiera eso (Experto CIDPA, 108).

Otra interpretación es que la actitud de consumidor es generada por los mismos programas que ofrece la política pública. En este caso no serían los jóvenes quienes se comportan como clientes frente a la política pública, sino que es la política pública misma la que genera clientes debido a la falta de coordinación entre los programas que ofrece (por ejemplo a nivel de la educación).

Ahora, cada programa buscaba su propio cliente, y generó también una política de clientelismo, y en ese sentido yo diría que el colegio y los estudiantes entraron en una lógica de cierto clientelismo. Es decir la pregunta ¿qué le trae este programa? Existía, esto si el programa trae consigo instalación de computadores, financiamiento para las acles, esta cantidad de libros, etc. [...] Entonces se generan muchos programas que el centro decía que era más el programa que la escuela, y por lo tanto los jóvenes también se vincularon con el programa en una lógica muy de clientelismo (Expertos CEJU, 47).

Por otro lado ¿qué otra cosa podemos esperar? ¿Acaso los jóvenes no han sido socializados en un contexto en donde el mercado funciona como mecanismo integrador (Sandoval, 2002) y en donde el Estado no es una figura central en la provisión de servicios públicos para la población joven, sino más bien una alternativa entre varias otras que ofrecen recursos para construir las trayectorias juveniles? 
En consideración de lo anterior, probablemente no sea posible hablar de la existencia de una oposición entre el ser actor y el ser cliente, sino que haya que reformular la cuestión de manera de visualizar a los jóvenes como consumidores y actores al mismo tiempo. Dicho de otra manera, comportarse como un consumidor puede entenderse como una nueva forma de agencia que permite a los jóvenes actuar y conseguir sus objetivos de acuerdo a las condiciones de su medio.

Es decir, yo creo que, en esta cosa erróneamente de creer que el joven no se da cuenta, pero sí. El joven es mucho más consciente de muchas cosas, sabe también aprovechar las oportunidades que se generan, saben que si hay financiamiento para esto, van a presentar proyectos para ellos etc. Es decir, por ejemplo, este juego que hay en el mundo actual juvenil, siento que el centro de alumnos no es lo más representativo, siento que el centro de alumnos en el fondo tiene que obedecer con la autoridad interna, etc., pero también sé que mediante el centro de alumnos yo puedo recibir recursos. Son cosas que lo colocan en una situación ambigua, lo mismo pasa con la federación de estudiantes universitarios. Es decir, recuerdo una época en mi juventud en donde se incentivó que las federaciones universitarias tuvieran personalidad jurídica, para poder recibir financiamiento del Estado. El problema estaba de que si llamaban a una marcha, y se provocaban destrozos, les llegaba también a ellos, entonces ese tipo de cosas lo hacen mas complejo. Pero yo creo que sí saben, y se relacionan con la política (Expertos CEJU, 48).

\section{REFLEXIONES FINALES}

Las políticas de juventud en Chile parecieran estar cada vez más distantes de quien es su población objetivo: los jóvenes. A pesar de los importantes avances alcanzados en orden a estudiar las realidades juveniles y a desarrollar una institucionalidad a favor de la población joven, sigue primando en el discurso público un concepto de juventud acotado y adultocéntrico. Los jóvenes son vistos principalmente como un grupo-problema (cf. IARD, 2001), frente al cual se tienden a elaborar e implementar políticas públicas orientadas a su control, generalmente vía el sistema educacional. Dentro de este marco el énfasis en las actividades de recreación por sobre otro tipo de actividades programáticas refuerza la idea de que los jóvenes no se encuentran aún en condiciones de asumir responsabilidades, lo que termina por situarlos en una condición de dependencia con respecto a los adultos. De lo 
anterior deriva una política pública que tiende a ver a los jóvenes como receptores pasivos, lo que fomenta su infantilización en vez de promover su autonomización y su formación como sujetos de derecho.

Se vuelve entonces un desafío central para las políticas públicas lograr construir un cuadro más complejo y menos lineal de las realidades juveniles, lo que hace necesario trascender la mirada de la juventud como un grupo inmaduro y problemático y aprender a concebir a los jóvenes como un recurso social a partir de su condición juvenil en el presente, lo que significa tomar en cuenta a los jóvenes en tanto jóvenes y no como potenciales adultos.

Lo anterior implica que las políticas públicas deben desarrollar iniciativas que no sólo aseguren la participación simbólica, sino también material de los jóvenes a la sociedad chilena. Esto supone necesariamente avanzar en políticas que logren reconocer y fomentar el desarrollo de autonomía de cada joven, lo que no sólo abarca la promoción de su educación escolar, la participación social en diversas asociaciones o la oferta de diversas actividades de tiempo libre, sino que también pasa por el fomento de una independencia material a través de una facilitación de la inserción laboral. Este último aspecto ha sido más bien descuidado por las políticas de juventud bajo la creencia de que juventud y educación conforman una unidad y de que los jóvenes deben prepararse para una vida adulta que será más adelante, pero no ahora. La incertidumbre de la época actual nos obliga a abandonar esta concepción lineal de las trayectorias biográficas y a reforzar la autonomía de los jóvenes en todos los sentidos incluyendo su independencia material. Creemos que será esto lo que finalmente permitirá una emancipación efectiva del control adulto y el empoderamiento del mundo juvenil. 


\section{REFERENCIAS BIBLIOGRÁFICAS}

BAEZA, JORGE (2006): «Demandas y organización de los estudiantes secundarios: una lectura sociológica más allá de fronteras y análisis coyunturales». Revista Electrónica Latinoamericana de Estudios sobre Juventud, Año 2, $\mathrm{N}^{\circ} 3$.

BENDiT, RENÉ. (2006): "Youth Sociology and Comparative Analysis in the European Union Member States». Papers 79. Barcelona: UAB.

Biggart, A., R. Bendit, D. CAIRns, K. Hein y S. MÖRCH (2002): Families and Transitions in Europe: State of the Art Report. Coleraine: University of Ulster.

CÁRDENAS, ANA (2009): «Transiciones en transición: Estado y jóvenes en Chile». En: Chile 2008: percepciones y actitudes sociales. $4^{\circ}$ Informe de Encuesta Nacional ICSO-UDP. Santiago: UDP.

CONTRERAS, DANIEL (2001): «Política social de juventud: ¿excluir o integrar a qué?». Última Década $\mathrm{N}^{\circ} 14$. Viña del Mar: Ediciones CIDPA.

DÁvILA, OsCAR (2008): «Institucionalidad y políticas de juventud». Revista Observatorio de Juventud N¹7. Santiago: INJUV. (2000): «Política pública e institucionalidad en juventud». Última Década $\mathrm{N}^{\circ} 12$. Viña del Mar: Ediciones CIDPA.

- (1999): «Políticas sociales, jóvenes y Estado: o el síndrome del padre ausente». Última Década N¹1. Viña del Mar: Ediciones CIDPA.

GOICOVIC, IGOR (2000): «Del control social a la política social. La conflictiva relación entre los jóvenes populares y el Estado en la historia de Chile». Última Década N¹2. Viña del Mar: Ediciones CIDPA.

IARD (2001): Study on the State of Young People and Youth Policy in Europe. Final Reports, Vol. 1: Executive Summary and Comparative Reports.

IGLESIS, ANDREA (2005): «Más derechos juveniles... Más seguridad, más integración, más democracia». Revista Electrónica Latinoamericana de Estudios sobre Juventud Año 1, $\mathrm{N}^{\circ} 1$.

- (2003): «Puntos ciegos de la política de juventud: ejes de referencia desde la práctica». En OsCAR DÁvILA (editor): Políticas públicas de juventud en América Latina: políticas locales. Viña del Mar: Ediciones CIDPA.

INJUV (2006): Segundo informe nacional de juventud. Condiciones de vida y políticas públicas de juventud desde la transición al bicentenario. Santiago: INJUV.

(2004): La integración social de los jóvenes en Chile (1994-2003). Individualización y estilos de vida de los jóvenes en la sociedad del riesgo. Santiago: INJUV.

KRAUSKOPF, DiNA (2008): «Avances y desafíos en la institucionalidad pública en juventud». Revista Observatorio de Juventud N¹7. Santiago: INJUV.

OIJ (2005): «Convención iberoamericana de derechos de los jóvenes». Madrid: OIJ. 
RAVINET, Eugenio (2008): «Políticas públicas en iberoamérica: el desafío hacia una visión común». Revista Observatorio de Juventud $\mathrm{N}^{\circ} 17$. Santiago: INJUV.

RODRÍGUEZ, ERNESTO (2003): «Políticas públicas de juventud en América Latina: empoderamiento de los jóvenes, enfoques integrados, gestión moderna y perspectiva generacional». Texto presentado en el seminario internacional «Producción de información y conocimiento para la formulación e implementación de políticas públicas de juventud», Manizales. En: www.urbared.ungs.edu.ar/textos/politicasdejuventud2.pdf.

Sánchez, Claudio (2000): «Una política de ciudad». Última Década №12. Viña del Mar: Ediciones CIDPA.

SANDOVAL, MARIO (2002): Jóvenes del siglo XXI. Sujetos y actores en una sociedad en cambio. Santiago: Ediciones UCSH.

Silva, Claudio (1999): «Ni héroes ni malvados, sólo jóvenes. Claves para iluminar la conversación sobre juventudes de los noventa». Última Década $\mathrm{N}^{\circ} 11$. Viña del Mar: Ediciones CIDPA.

WALTHER, ANDREAS y BARBARA STAUBER (eds.) (2002): Misleading Trajectories. Integration Policies for Young Adults in Europe? A Publication of the European Group for Integrated Social Research (EGRIS). Opladen: Leske+Budrich. 